\title{
Adaptive Filtering of Evoked Potentials With Radial-Basis-Function Neural Network Prefilter
}

\author{
Wei Qiu, Member, IEEE, Kenneth S. M. Fung, Francis H. Y. Chan*, Member, IEEE, F. K. Lam, Paul W. F. Poon, \\ and Roger P. Hamernik
}

\begin{abstract}
Evoked potentials (EPs) are time-varying signals typically buried in relatively large background noise. To extract the EP more effectively from noise, we had previously developed an approach using an adaptive signal enhancer (ASE) (Chen et al., 1995). ASE requires a proper reference input signal for its optimal performance. Ensemble- and moving window-averages were formerly used with good results. In this paper, we present a new method to provide even more effective reference inputs for the ASE. Specifically, a Gaussian radial basis function neural network (RBFNN) was used to preprocess raw EP signals before serving as the reference input. Since the RBFNN has built-in nonlinear activation functions that enable it to closely fit any function mapping, the output of RBFNN can effectively track the signal variations of EP. Results confirmed the superior performance of ASE with RBFNN over the previous method.
\end{abstract}

Index Terms-Adaptive signal enhancer (ASE), evoked potential, radial basis function neural network (RBFNN), SNR.

\section{INTRODUCTION}

$\mathbf{E}$ VOKED potentials (EPs) represent the gross electrical activity of specific regions of the brain usually resulting from sensory stimulation. Like many neural signals, the measurement of EPs is corrupted by noise as a result of the on-going activity of other brain cells known as electroencephalographic (EEG) activity. The traditional method of ensemble averaging (EA) has been widely used to extract EP from a noisy background. However, a number of studies showed that EPs are nonstationary and, therefore, have characteristics that vary across stimulus trials [14]-[18]. EA methods may, therefore, fail to track trial-to-trial variations both in latency and amplitude.

Adaptive filtering has been widely used in biological signal estimation [2], [3], [5]-[7], [10]-[13]. The processing units in the conventional adaptive filter $(\mathrm{AF})$ are linear in nature. Adaptive signal enhancer (ASE) represents a special approach based

Manuscript received November 8, 2000; revised September 13, 2001. This work was supported in part by National Nature Science Foundation under Grant 69871011, in part by the Research Grant Council under Grant HKU 553/96M, Grant HKU 7036/97E, and Grant CUHK 4260/99M, and in part by the Ministry of Education of Taiwan under Academic Excellence Grant 89-B-FA08-1-4. Asterisk indicates corresponding author.

W. Qiu and R. P. Hamernik are with the Auditory Research Laboratory, State University of New York, Plattsburgh, NY 12901 USA.

K. S. M. Fung and F. K. Lam are with the Department of Electrical and Electronic Engineering, the University of Hong Kong, Hong Kong.

*F. H. Y. Chan is with the Department of Electrical and Electronic Engineering, the University of Hong Kong, Pokfulam Road, Hong Kong (e-mail: fychan@eee.hku.hk).

P. W. F. Poon is with the Department of Physiology, College of Medicine, National Cheng Kung University, Taiwan.

Publisher Item Identifier S 0018-9294(02)01749-4. on AF. It allows tracking of the dynamic variations of EP and reduces the noise uncorrelated with the underlying signal. The performance of ASE greatly depends on its reference signal. Several reference signals have been tested. Vaz and Thakor [13] used a finite number of sine and cosine waves as the reference input in the time domain. It was assumed that the signal is well defined in each recurrence with a constant number of reference inputs. Laguna, et al. [10] designed the reference input as a unit impulse sequence synchronized with the beginning of each recurrence. This method takes advantage of the fact that EPs are responses that are time-locked to the stimulus. Mathematical proof shows that such a filter is equivalent to exponentially weighted averages. Qiu et al. [7] adopted a dynamic averaging window to obtain a more meaningful reference.

All of the methods discussed above basically involve linear operations. Obviously, the assumption of linearity for the nervous system may not be always valid. Since at the single cell level, it is well known that for identical stimuli, responses of central neurons show jitter in latency and responses may sometimes fail to show up. Taking the possible nonlinear nature of the system into consideration, neural networks that can intrinsically cater to both linear and nonlinear characteristics are potentially more appropriate. Recently, the use of neural networks with built-in nonlinear processing units has been growing in many areas of signal processing [8], [9], [19], [20]. In our previous work [9], a radial-basis-function neural network (RBFNN) was successfully used to estimate the EP. The structure of the RBFNN is relatively simple with an output node that has a linear-in-weight property. Therefore, it represents a good balance between performance and computational complexity. Its powerful modeling capability allows it to learn the local representation of any nonlinear function. It has been shown that RBFNN is capable of forming an arbitrarily close approximation to any continuous function [21]. In our previous work [9], RBFNN was utilized to extract an EP signal. It was assumed that EP responses could be modeled by a finite number of Gaussian radial-basis functions (RBFs) with their centers evenly distributed over time. The weights are adaptively determined by minimizing the variance of the error signal using the least mean square (LMS) algorithm. Since the RBFNN is dynamically adjusted to changes in EP, the nonstationary property of EP is catered to. Results of our previous work confirmed the good tracking ability of RBFNN on EP.

Ferrara and Widrow [3] proved that in estimating signals, the improvement in signal-to-noise ratio (SNR) at the ASE output is proportional to the SNR at the reference input. Therefore, the performance of ASE critically depends on its reference signal. 
The higher the correlation between the reference input and the desired signal, the better is the signal estimation. Since RBFNN can track signal variations with good SNR improvement [9], the output of RBFNN can form a better reference input for the ASE. In this paper, a new ASE approach with an RBF prefilter is proposed. With the RBF combined as the prefilter of ASE, a novel adaptive EP estimation scheme is achieved to out-perform the former algorithms that use RBF or conventional ASE. Simulation and real data analysis showed that this new method is effective in tracking EP variations across trials and allows fast EP measurement in many time-critical circumstances.

\section{MATERIALS AND METHODS}

\section{A. Principle of ASE}

Standard ASE has two inputs: the primary input that contains a signal $s_{0}$ plus noise $n_{0}$, and the reference input that contains a signal $s_{1}$, related to but not necessarily having the same waveform as $s_{0}$, plus a noise $n_{1}$. The noise $n_{0}$ and $n_{1}$ are assumed unrelated to each other and to both signals. The ASE iteratively adjusts its impulse response via an adaptive algorithm so that, after convergence, the difference between the filter output $y$ and desired response $d$ is minimized.

We used the well-established LMS algorithm for AF [1], [4]. Its recursive formula is

$$
w_{k+1}=w_{k}+2 \mu \varepsilon_{k} x_{k}
$$

where

$$
\varepsilon_{k}=d_{k}-y_{k}
$$

and

$$
y_{k}=X_{k}^{T} W_{k}=W_{k}^{T} X_{k} .
$$

The filter input vector is defined as

$$
X_{k}=\left[\begin{array}{lllll}
x_{k} & x_{k-1} & x_{k-2} & \cdots & x_{k-Q+1}
\end{array}\right]^{T} .
$$

The weight vector is defined as

$$
W_{k}=\left[\begin{array}{lllll}
w_{0 k} & w_{1 k} & w_{2 k} & \cdots & w_{(Q-1) k}
\end{array}\right]^{T}
$$

where $k$ is the time index, $Q$ the number of adaptive weights, and $\mu$ the factor that controls stability and the rate of convergence. Usually $\mu$ is governed by the following condition:

$$
0<\mu \ll \frac{1}{Q P_{i n}}
$$

where $P_{i n}$ is the power of the reference input. After convergence, the output of ASE can estimate the signal component of the primary input as long as $s_{1}$ is highly correlated with $s_{0}$. The SNR of the reference input should be larger than $0 \mathrm{~dB}$ to achieve good performance [3]. In this paper, we use RBFNN as a prefilter to get an even better reference input especially when SNR is low.

\section{B. Gaussian RBFNN for EP Prefilter}

The RBFNN is a multilayer feed-forward neural network consisting of an input layer of source nodes, a layer of nonlinear hidden units that operate as kernel nodes and an output layer of linear weights. In response to an input vector $\mathbf{X}$, the outputs, $h_{j}(\mathbf{X})$, of the hidden layer are linearly combined to form the network response that is processed with a desired response presented to the output layer. The weights are trained in a supervised fashion using an appropriate linear learning method (LMS algorithm). The LMS algorithm provides a bridge between linear adaptive algorithms and neural networks. Having $\mathrm{X}$ as an input, the following formula represents the output of a RBFNN that has $N$ hidden nodes:1

$$
y(\mathbf{X})=\sum_{j=1}^{N} w_{j} h_{j}(\mathbf{X}) .
$$

A radially symmetric Gaussian RBF was adopted as the activation function for the hidden nodes [9]. The response of RBFNN is related to the distance between the input and the centroid associated with the basis function.

The LMS algorithm was used to optimize the weight vector adaptively [1]. Assuming background noises are white and unrelated to the EP signal, the improvement of SNR at steady state (i.e., after several time constants) is described as [9]

$$
\Delta \mathrm{SNR} \approx \frac{\mathrm{N}-1}{2 \mu_{\mathrm{rbf}}^{2} \pi^{3 / 2}(\mathrm{M}-1) \beta^{3}}
$$

where $M$ is the number of data points in each recorded trial of the EP signal, $\mu_{\mathrm{rbf}}$ is the step size parameter and $\beta$ was set to 0.8 , an experimentally obtained value. As can be seen from (8), the improvement of the SNR has an upper bound since we have to make a trade-off between the convergence rate $\mu$ and the number of hidden nodes $N$. RBFNN, therefore, tracks the trial-to-trial changes in EP by the nonlinear RBFs that fit dynamically the EP variations via an adaptive algorithm. Additional details on RBFNN are presented in a previous paper [9].

\section{ASE With RBF Prefilter}

As described above, in order to establish good tracking ability, the quality of the reference signal is the most critical factor. Since EP is heavily contaminated with background EEG, it is hard to obtain a good reference signal. In our previous work [5], we used a moving window average (MWA) and ensemble average (EA) to construct the reference input for the ASE. The improvement of EA on SNR is proportional to the square root of the number of trials $(m)$ being averaged, i.e., $\Delta S N R \sim \sqrt{m}$. Assuming a white background noise, and raw EP signals with a SNR of $-10 \mathrm{~dB}$, m needs to exceed 100 for the SNR to be improved to $0 \mathrm{~dB}$. With $\mu_{\mathrm{rbf}}=0.1$, $N=10, M=100$, and $\beta=0.8$, in the RBFNN, the SNR can theoretically be improved as much as $2 \mathrm{~dB}$. This means that each single output of RBFNN is sufficiently good enough to be taken as the reference input.

Fig. 1 shows the structure of the new adaptive EP signal enhancer. The EP preenhanced by the RBFNN is given by the weighted sum of $N$ evenly distributed RBFs $\Phi_{j}(j=1$ to $N)$, $r_{i}(k)=\sum_{j=1}^{N} w_{j} \varphi_{j}(k)$ where $i$ and $k$ denote, respectively, the trial number and time index corresponding to the $i$ th stimulus. Assuming a null mean in the background noise which is statistically uncorrelated with the underlying EP signal component $s_{i}(k)$, then $r_{i}(k)$ tracks the underlying EP using LMS al- 


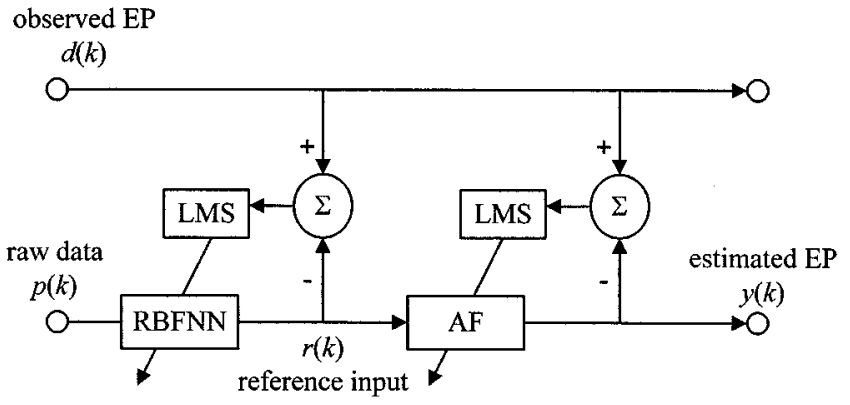

Fig. 1. The schematic diagram of ASE with RBFNN as a prefilter.

gorithm by minimizing the error signal $e_{i}(k)=d_{i}(k)-r_{i}(k)$, where $d_{i}(k)$ is the recorded EP. Since $r_{i}(k)$ has a high SNR and is correlated with $s_{i}(k)$, it is a desirable reference signal.

\section{RESULTS}

In our previous work [5], an ASE, with MWA as a reference input, can achieve single EP tracking at SNRs of raw data as low as $-10 \mathrm{~dB}$. With lower SNRs (e.g., $-20 \mathrm{~dB}$ ), tracking degrades significantly because more trials are required for averaging with the moving window. In such cases, EA is used to obtain the reference input. Since EA averages all EP records before the ASE processing, it is no longer a real-time method. Moreover, if the EP signal varies greatly across trials, the reference input obtained with EA will result in large distortions affecting the performance of ASE.

\section{A. Comparison in Performance}

Before applying our new method to real EP estimation, computer simulations were carried out. A realistic brainstem auditory EP (BAEP) obtained from a human by averaging 2000 response trials was used as the signal in the simulation. To this BAEP, simulated EEG noise is added before its presentation as the primary input of ASE. The SNR of the primary input is set at $-20 \mathrm{~dB}$. The simulated on-going EEG is produced by the following formula [5]:

$$
\mathrm{EEG}_{\mathbf{n}}=\frac{1}{429} \sum_{\mathrm{l}=-5}^{5} \mathrm{~h}_{\mathbf{l}} \mathrm{r}_{\mathrm{n}+\mathrm{l}}
$$

where $r_{n}$ is a driven white noise process with a Gaussian distribution and the coefficients $h_{l}$ have the following values:

$$
\begin{aligned}
& h_{l}=h_{-l}, \quad h_{0}=89, \quad h_{1}=84, \quad h_{2}=69, \\
& h_{3}=44, \quad h_{4}=9, \quad h_{5}=-36 \text {. }
\end{aligned}
$$

The data set contains 1000 trials and each trial has 90 data points.

The relative mean square error (MSE) was used to measure the effectiveness of the method. The MSE is defined as

$$
\mathrm{MSE}=\frac{\mathrm{E}\left[(\mathrm{s}-\mathrm{y})^{2}\right]}{\mathrm{E}\left[\mathrm{s}^{2}\right]}
$$

where $y$ is the output of the ASE and $s$ is the signal to be detected. The performance of ASE was compared with various kinds of reference inputs with or without RBFNN. The different reference inputs include the following.

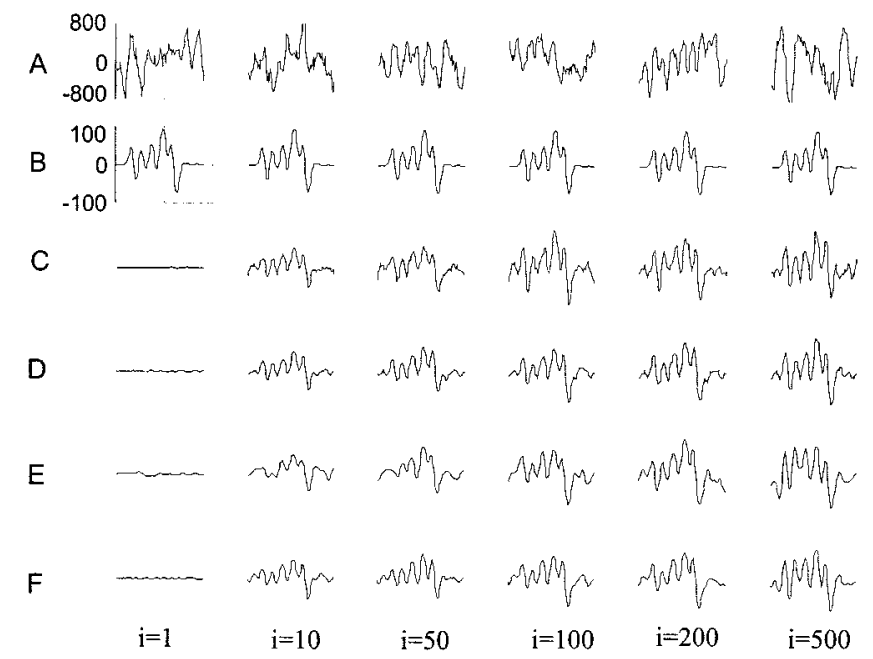

Fig. 2. Adaptive procedures of ASE ( $i$ indicates trial number). (A) Primary inputs of ASE (SNR $=-20 \mathrm{~dB}$ ). (B) Underlying simulated BAEP signals. (C) Some results of ASE with MWA. (D) Some estimated BAEP signals by ASE with EA. (E) Results of RBFNN. (F) Adaptive processing results of ASE with RBFNN.

A) MWA (real-time mode).

The primary input is

$$
d_{i}=\operatorname{trial}(i) \quad i=q+1, q+2, \ldots, L .
$$

The reference input is

$$
x_{i}=\frac{1}{q} \sum_{j=i=q+1}^{i-1} \operatorname{trial}(j)
$$

where $q$ is the window length.

B) EA (nonreal-time mode).

The primary input is

$$
d_{i}=\operatorname{trial}(i) \quad i=1,2,3, \ldots, L .
$$

The reference input is

$$
x_{i}=\frac{1}{L-1} \sum_{j=1(j \neq i)}^{L} \operatorname{trial}(j) .
$$

C) $\quad$ RBF prefilter.

The primary input is

$$
d_{i}=\operatorname{trial}(i) \quad i=p+1, p+2, \ldots, L .
$$

The reference input is

$$
x_{i}=\frac{1}{p} \sum_{j=i-p+1}^{i-1} \operatorname{rbfnn}(j) .
$$

The value of the convergence rate was determined from

$$
\mu=\frac{\alpha}{Q P_{\text {in }}}, \quad \alpha \ll 1
$$

where $P_{i n}$ is the mean power of the reference input. In the three ASE structures, the constant $\alpha$ was set at 0.05 . MWA could be 


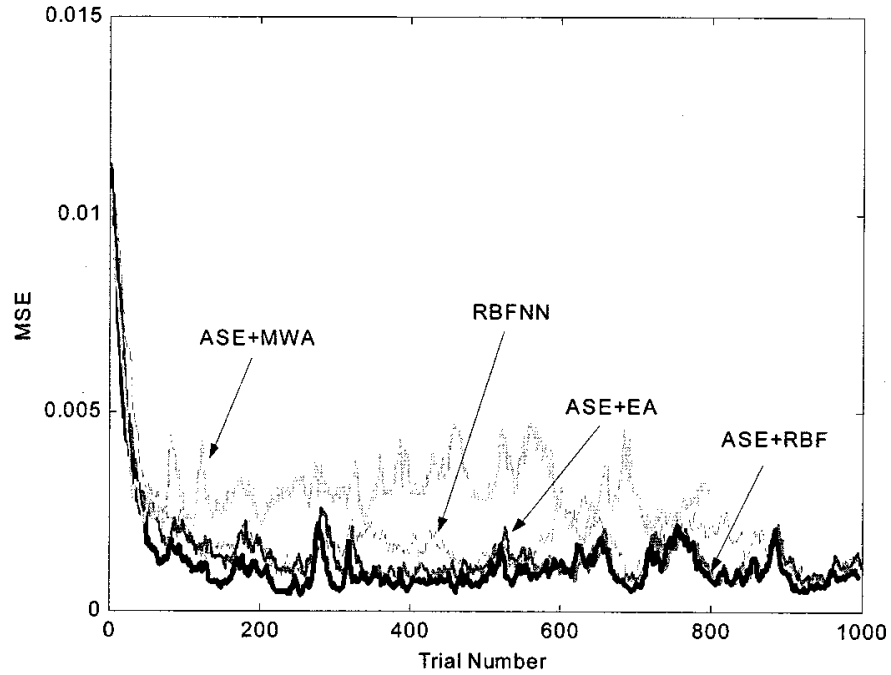

Fig. 3. A comparison of the four ASE approaches based on MSEs.

used with RBFNN prefilter to further improve the SNR. However, since RBFNN achieved good improvement in SNR, a small window length $p$ was desirable. In our case, $p$ was selected as three. In (16), the function $r b f n n(j)$ represents the output of RBFNN at the $j$ th trial. The parameters of RBFNN were set as follows: $\mu_{\mathrm{rbf}}=0.05, N=30, M=90$, and $\beta=0.8$.

Fig. 2 illustrates the adaptive procedure of ASE in the example. Fig. 2(A) is the primary input consisting of a signal with additive EEG noise. Fig. 2(B) shows the underlying BAEP signals. Note that the amplitude scale of the primary input of ASE is $[-800,800]$ while that of BAEP signal is $[-100$, 100]. The scales in the figure caption are relative scales. In the simulation, the scale only displays the relative amplitude of the simulated EP recordings and EP signal. In real data analysis, the relative scale could be easily transferred into a real scale (such as voltage values) according to analog-to-digital specifications. A relative scale has been used throughout this paper. The results of ASE using MWA $(q=200)$ and EA are shown in Fig. 2(C) and (D), respectively. Fig. 2(E) shows some results of RBFNN and Fig. 2(F) the results of ASE with RBFNN prefilter $(p=3)$. Since EP signal remains the same across trials, all of the above four methods were found to detect the EP signal reasonably well. To compare their performance more closely, their MSEs were calculated. Fig. 3 shows the variation of MSE against trial number. To compare the convergence rate, we moved the MSE curve of ASE with MWA to the staring point of the other curves. From Fig. 3, it is clear that the convergence rates are similar for the four algorithms. Actually, the performances of the algorithms are also similar except for ASE with MWA. The poorer performance of ASE with MWA comes from the poor SNR of the reference input since averaging of 200 trials is not large enough to make a sufficiently good reference. An increased window length improves the SNR at the expense of signal tracking ability (see Section III-B). The limiting case of MWA in this simulation is actually EA. ASE with EA can provide good EP estimation mainly because of a constant EP signal across trials. The performance of ASE with RBFNN is a slightly better than RFBNN alone or ASE with EA. However, the difference is not significant.

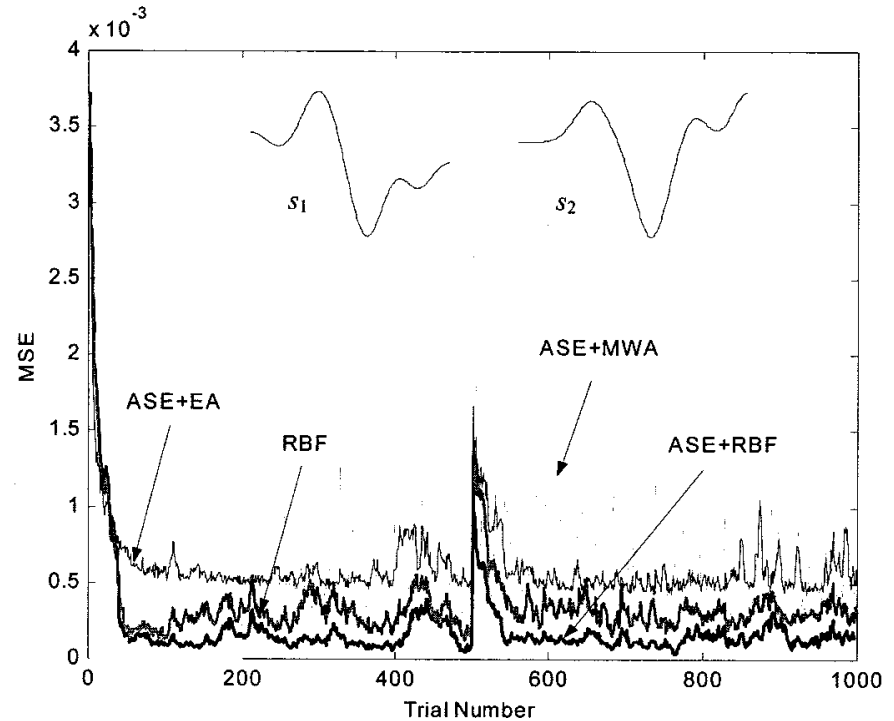

Fig. 4. Error curves for RBFNN, ASE with MWA, ASE with EA, and ASE with RBFNN algorithms in an abrupt EP changing simulation. Insets are two simulated underlying VEP signal, $s_{1}$ is the signal in first 500 trials and $s_{2}$ is the signal in last 500 trials.

\section{B. Performance of Tracking EP Signal Variation}

The performance of ASE with RBFNN prefilter in tracking EP signal variations across trials is investigated in this section. A data set is first generated to simulate abrupt changes of EP waveform. The simulated signals are the visual EPs (VEPs) obtained in a normal human subject. Simulated EEG noise from (9) is superimposed on the underlying signal to give the simulated VEP a SNR of $-20 \mathrm{~dB}$. The data set consists of 1000 trials. The first 500 trials adopt signal $s_{1}$ (refer to the insets in Fig. 4) as the underlying signal. Then there is an abrupt change with the remaining 500 trials adopting a different underlying signal, $s_{2}$. The four algorithms of EP extraction described in Section III-A are again used and their tracking ability compared.

Fig. 4 shows the error curves. It is clear that the ASE with RBFNN as prefilter can best track the signal variations. MSE of ASE with EA is significantly larger than that of either RBFNN alone or ASE with RBFNN. Comparing results of ASE with either EA or MWA, we noticed that although the result with MWA looks noisier than EA, in many trials its errors appear lower. This can be explained by a higher correlation between the signal component in the reference input and that in the raw data for the case of MWA, but the SNR in the reference input is also lower. For the EA as reference input of ASE, if the signal waveform changes significantly across trials, EA will degrade the correlation of the signal components between the reference input and the primary input. Another interesting point is the poorer performance of ASE with MWA at the transitional period (from trial 501 to 700 , at $q=200$ ) when the signal in the reference input changes from $s_{1}$ to $s_{2}$. The error at that duration is higher than other periods. Since both $s_{1}$ and $s_{2}$ signals are in the MWA window, the mixture of two signal components may cause less correlation between the reference and the primary inputs.

From the above, it was found that ASE with MWA might not be suitable for signal tracking under poor SNR conditions. Since a poor SNR in the raw data requires a longer window to form 

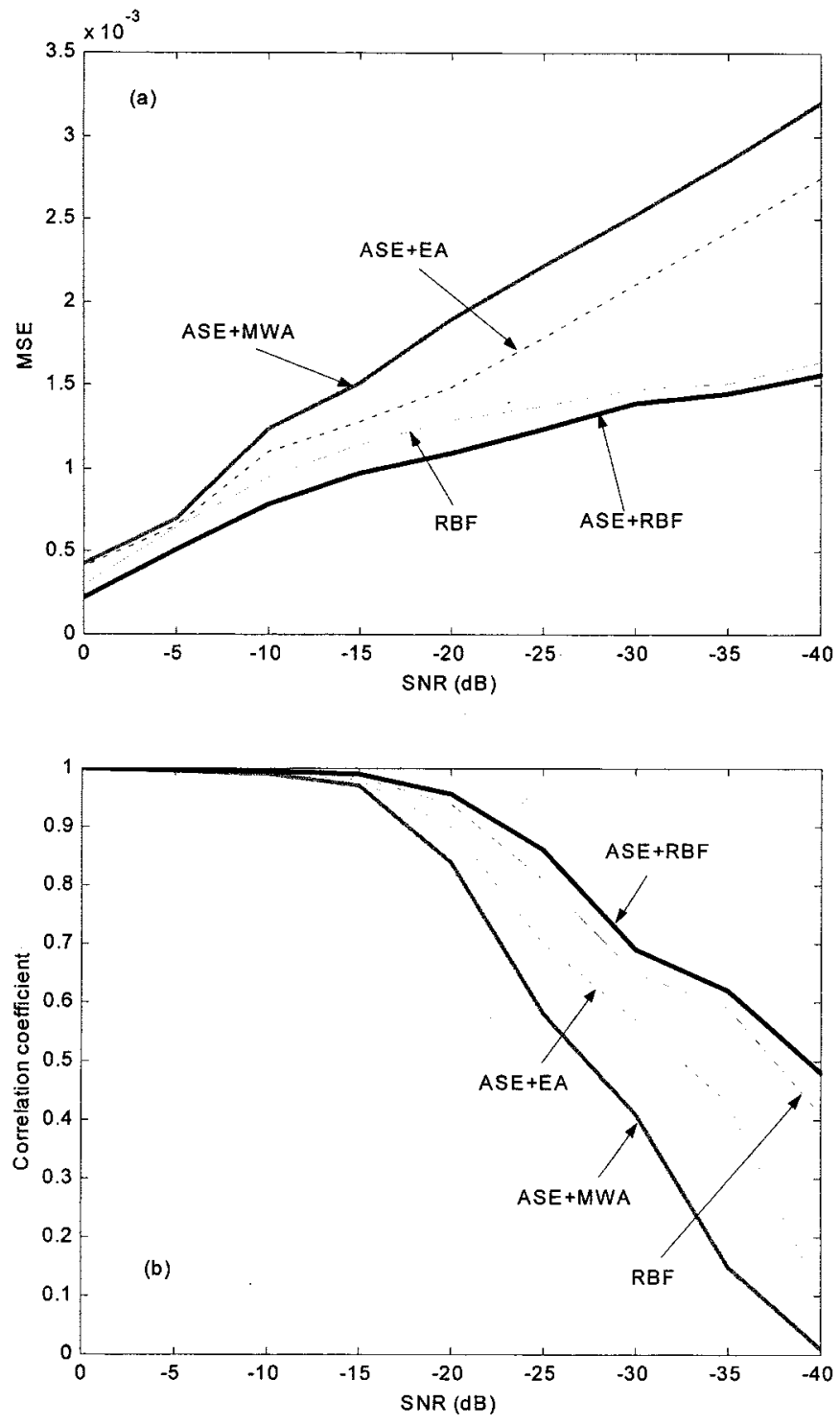

Fig. 5. Performance comparison for the four algorithms at different noise levels. (a) Average MSEs. (b) Correlation coefficients for the four algorithms.

a reference input with a good SNR. However, a longer window will degrade the ability of EP tracking. Therefore, ASE with EA may not be good enough to track signal variations since EA will lower the signal correlation between the reference input and the primary input. It is difficult to find an optimum window of MWA since it is usually not possible to get a priori knowledge on the variation of EP signal and SNR in a raw recording. The length of window is, therefore, estimated on the basis of experimental results. As discussed in a previous paper [9], RBFNN is a method that is suitable for estimating EP and its transient changes that are not known a priori. The above simulation further illustrates this point by showing the better performance of ASE with RBFNN as a prefilter.

\section{Comparison on Performance at Different Noise Levels}

The simulated signal and EEG noise were the same as Section III-A. Different SNR for simulated EP recordings were created to compare filter performance at different noise levels. The SNR range was varied from $-40 \mathrm{~dB}$ to $0 \mathrm{~dB}$ in 5-dB increments.

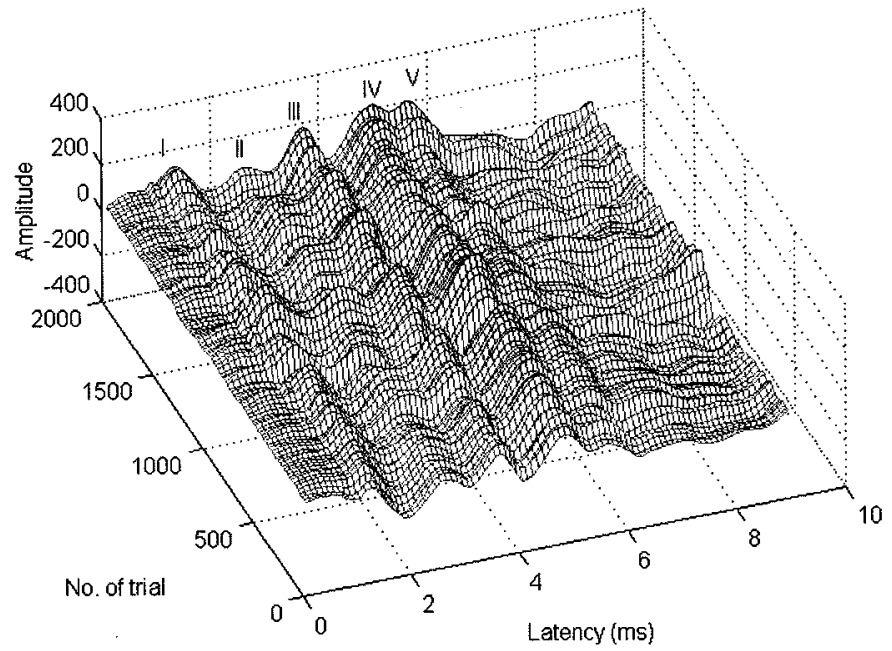

Fig. 6. Isometric view of BAEP traces from a human subject.

In this case, the EP signal was deterministic across trials. The number of trials was 1000 . Four filtering algorithms were used to process the simulated recordings. MSEs were calculated trial by trial. The last $200 \mathrm{MSEs}$ of were averaged to get an averaged MSE at different noise levels using each algorithm. For ASE with MWA algorithms, the length of the moving window is set from 100 to 500 in increments of 50 trials at each noise level and the best result is shown in Fig. 5. The parameter $p$ for ASE with RBFNN was varied from 5 to 30 in increments of five and the best result is shown in Fig. 5. Fig. 5(a) gives the comparison in performance by averaged MSE at different noise levels. It is clear that MSEs of both RBF and ASE+RBF are smaller than that of ASE with EA and ASE with MWA at all the noise levels. When the SNR becomes poorer MSE increases very quickly with ASE+EA and ASE+MWA while the performance of RBFNN and ASE with RBFNN is relatively better. To quantitatively assess filter performance, correlation coefficients were calculated between the original signal and the averaged reconstructed signal, which was formed from averaging the last 200 processed trials. Fig. 5(b) shows the correlation coefficients at different noise levels. The distortions become significant for ASE with MWA and ASE with EA when SNR is below - $20 \mathrm{~dB}$. The performance of RBFNN and ASE with RBFNN remains better. However, if the SNR is very poor, (i.e., $-40 \mathrm{~dB}$ or less) even RBFNN and ASE with RBFNN introduce marked distortions. For those recordings with very poor SNRs more trials are needed to achieve more reliable estimates of the signal.

\section{REAL EP DATA ACQUISITION AND PROCESSING}

In this section, ASE with RBFNN is used to estimate BAEP from actual recordings of a human subject and to monitor somotosensory evoked potential (SEP) changes from a rabbit.

\section{A. Human BAEP Estimation}

The click stimulus used to evoke the BAEP was $0.1 \mathrm{~ms}$ in duration, and delivered at a rate of 10/s, with a peak level of 100-dB SPL. The EP response was sampled at $10 \mathrm{kHz}$. The first $9 \mathrm{~ms}$ of the poststimulus response (corresponding to 90 data points) 

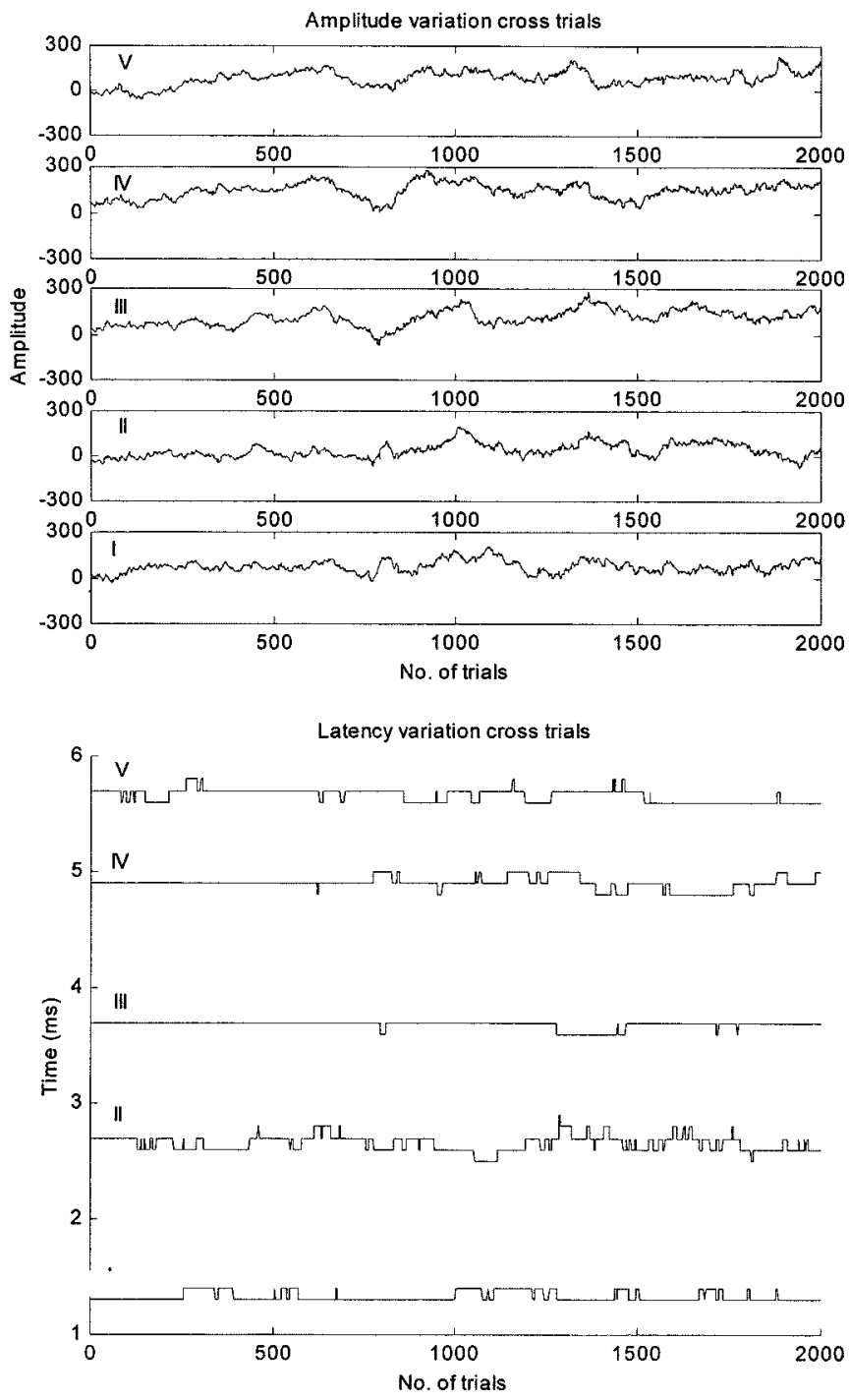

Fig. 7. Trace of the amplitude and latency variation of BAEP from a human subject.

was used for BAEP estimation. Conventional EP recording procedures, such as electrical and acoustic shielding, were used to reduce interference and noise.

A total of 2000 trials of BAEP were recorded from a 25-year-old subject. For ASE the step size $\mu$ was calculated according to (17) with $\alpha=0.05$. For RBFNN prefilter, the following parameters were set: $\mu_{\mathrm{rbf}}=0.05, N=30, M=$ 90 , and $\beta=0.8$. Fig. 6 shows some isometric views of BAEP across trials. It is clear that the BAEP signal varies in both amplitude and latency from trial to trial. Normally, there are five peaks in the early BEAP (see marks in the figures). Using the ASE with RBFNN approach the time and amplitude variations of the five peaks can be tracked across trials. Fig. 7 shows traces of the amplitude and latency variation of BAEP from a human subject. The variations of latency are relatively small and slow, while the peak amplitudes show larger variations across trials.

\section{B. Experimental Spinal Cord Monitoring Study}

The study subject was a mature rabbit. The purpose of this experiment was to study an ischemia induced spinal cord in-

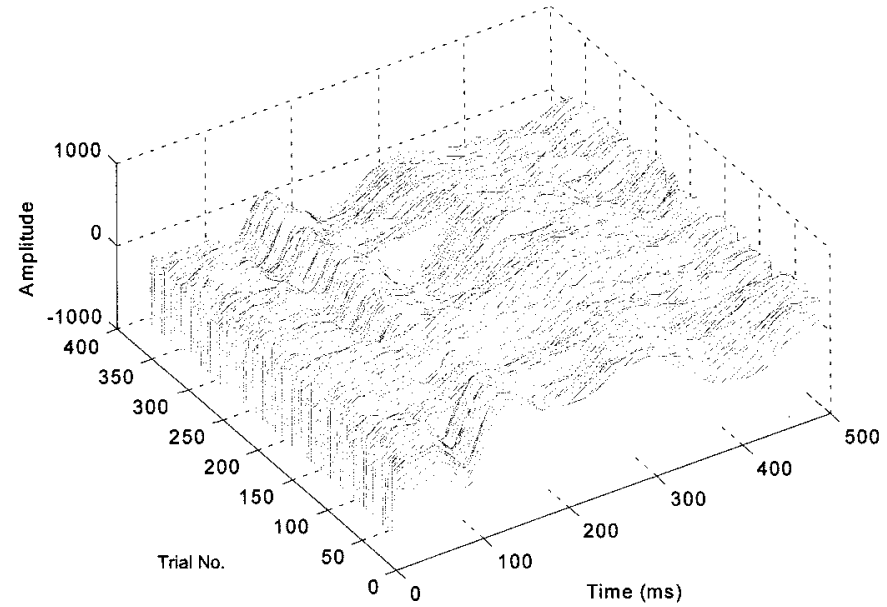

Fig. 8. ASE+RBFNN analysis of SEP in an ischemia spinal cord injury model. The figure presents seven sets of SEP traces, each set consisting of 51 separate traces. The SEPs are presented consecutively as recorded prior to injury (trace number 1 to 51), at the two recording periods during occlusion (trace number 52 to 153 ) and at the four postocclusion intervals (trace number 154 to 357$)$.

jury model. Anesthesia was initially induced with $1.5 \%$ sodium pentobarbital injection $(1.5 \mathrm{ml} / \mathrm{kg}$, intravenously). Additional doses were given as necessary. After anesthesia, a pair of SEP recording electrodes was inserted to the animal's skull. A needle stimulus electrode was applied to the hind paw. Baseline SEP was recorded prior to injury. The infrarenal abdominal aorta was then occluded. After 40 min the clamp on the aorta was removed. SEPs were recorded at 15 and 30 min after occlusion, and 20,40,60, and $120 \mathrm{~min}$ after disocclusion. All the data were recorded using an $\mathrm{ADC}$ board with a resolution of 8 bits. The sampling rate was $5 \mathrm{kHz}$.

Fig. 8 shows the SEP trace variations for all the trials recorded before, during and after occlusion using the ASE with RBFNN algorithm. The figure presents seven sets of SEP traces, each set consisting of 51 separate traces. The SEPs are presented consecutively as recorded prior to injury (trace number 1 to 51 ), at the two recording periods during occlusion (trace number 52 to 153) and at the four postocclusion intervals (trace number 154 to 357). The SEP waveform prior to injury has a prominent peak between 100 and $200 \mathrm{~ms}$. At the 15 and 30 min during occlusion-recording interval (trace number 52 to 153), the peak clearly disappeared. After the occlusion is removed the peak gradually returns to its prior to injury level (trace \#154 through 357).

\section{CONCLUSION}

This research shows that ASE with RBFNN as a prefilter is applicable to the real-time detection of EP signals. We have described a method of ASE and have used it to determine the BAEP signal in human subjects in [7]. The key to ASE for tracking signal variations is the choice of the reference input. It has been shown [3] that the improvement in SNR at the output of ASE is proportional to the SNR at the reference input. The signal component of the reference input should be highly correlated with that of the primary input, although not necessarily having the identical waveform. A MWA is a simple method for 
obtaining a good reference input for processing event-related bioelectric signals. However, when SNR of raw recordings is low (i.e., $-20 \mathrm{~dB}$ or less), the window should be long enough to obtain a reasonable SNR in the reference input. Unfortunately, a long window length will degrade the tracking ability of ASE. Simulation results showed that MWA is not suitable for constructing the reference input needed for the ASE to track the signal variation under very poor SNR conditions. EA is an alternative method that can be used to obtain a reference input with a high SNR. When the raw SNR is low, EA needs hundreds or thousands of trials before ASE can be effectively used to estimate the EP signal. Thus, it is no longer a real-time approach and can only be used when the EP does not change significantly across trials.

A RBFNN consists of an input of source nodes, a single hidden layer of nonlinear processing units, and an output layer of linear weights. This structure makes RBFNN a very powerful tool for solving both linear and nonlinear problems. Our previous work [9] has shown that RBFNN is a very good approach for tracking signal changes. In this paper, the RBFNN is used as a special prefilter to form the reference input of ASE. Simulation studies showed that the performance of ASE with RBFNN is better than both RBFNN and ASE with MWA or EA under different SNR conditions.

The clinical relevance of the variation in response across stimulus trials is not clear at this stage. Since the present study only concentrates on implementing an effective method for signal tracking. But one is tempted to speculate that at least under some conditions tracking EP changes could be important: e.g., for patients under critical care when their physiological conditions may vary with time, or when the status of the patient may vary under the influence of drugs like alcohol. A simple measurement of EP amplitude or latency may not be sensitive enough to reveal important changes. Early alarm systems may be designed based on the findings of abnormal response variations. In addition, EP variations over time could be important in assessing abnormal functions of the brain especially related to attentional deficits in psychiatry.

\section{ACKNOWLEDGMENT}

The authors would like to thank Dr. Y. Hu for providing rabbit SEP recordings.

\section{REFERENCES}

[1] B. Widrow and S. D. Stearns, Adaptive Signal Processing. Englewood Cliffs, NJ: Prentice-Hall, 1985.

[2] B. Widrow, J. R. Glover, Jr., J. M. Mccool, J. Kaunitz, C. S. Williams, R. H. Hearn, J. R. Zeidler, E. Dong, Jr., and R. C. Goodlin, "Adaptive noise cancelling: Principles and applications," Proc. IEEE, vol. 63, pp. 1692-1716, Dec. 1975

[3] E. R. Ferrara and B. Widrow, "Multichannel adaptive filtering for signal enhancement," IEEE Trans. Acoust., Speech Signal Processing, vol ASSP-29, pp. 766-770, 1981.

[4] S. Haykin, Adaptive Filter Theory. Englewood Cliffs, NJ: PrenticeHall, 1986.

[5] F. H. Y. Chan, F. K. Lam, P. W. F. Poon, and W. Qiu, "Detection of brainstem auditory evoked potential by adaptive filtering," Med. Biol. Eng. Comput., vol. 33, pp. 69-75, 1995.

[6] F. H. Y. Chan, W. Qiu, F. K. Lam, P. W. F. Poon, and M. K. Lam, "Evoked potential estimation using modified time sequenced adaptive filter," Med. Biol. Eng. Comput., vol. 36, pp. 407-414, 1998.
[7] W. Qiu, F. H. Y. Chan, F. K. Lam, and P. W. F. Poon, "Brainstem auditory evoked potential measurement using adaptive signal enhancement," Australasian Phys. Eng. Sci. in Med., vol. 17, no. 3, pp. 131-135, 1994.

[8] K. S. M. Fung, F. H. Y. Chan, F. K. Lam, J. G. Liu, and P. W. F. Poon, "Visual evoked potential enhancement by an artificial neural network filter," Biomed. Mater. Eng., vol. 6, pp. 1-13, 1996.

[9] K. S. M. Fung, F. H. Y. Chan, F. K. Lam, and P. W. F. Poon, “A tracing evoked potential estimator," Med. Biol. Eng. Comput., vol. 37, pp. 218-227, 1999.

[10] P. Laguna, R. Jane, O. Meste, P. W. Poon, P. Caminal, H. Rix, and N. V. Thakor, "Adaptive filter for event-related bioelectric signals using an impulse correlated reference input: Comparison with signal averaging techniques," IEEE Trans. Biomed. Eng., vol. 39, pp. 1032-1044, 1992.

[11] P. G. Madhavan, "Minimal repetition evoked potentials by modified adaptive line enhancement," IEEE Trans. Biomed. Eng., vol. 39, pp. 760-764, July 1992.

[12] N. V. Thakor, "Adaptive filtering of evoked potentials," IEEE Trans. Biomed. Eng., vol. BME-34, pp. 6-12, Jan. 1987.

[13] C. A. Vaz and N. V. Thakor, "Adaptive Fourier estimation of time-varying evoked potentials," IEEE Trans. Biomed. Eng., vol. 36, pp. $448-455,1989$.

[14] T. Harmony, Neurometric Assessment of Brain Dysfunction in Neurological Patients. Hillsdale, NJ: Lawrence Erlbaum, 1984.

[15] C. D. McGillem and J. I. Aunon, "Methods of analysis of brain electrical and magnetic signals," in EEG Handbook of Electroencephalography and Clinical Neurophysiology, A. S. Gevins and A. Remond, Eds. Amsterdam, The Netherlands: Elsevier, 1987, ch. 5.

[16] D. Regan, Human Brain Electrophysiology: Evoked Potentials and Evoked Magnetic Fields in Science and Medicine. New York: Elsevier, 1989.

[17] S. Nishida, M. Nakamura, and H. Shibasaki, "Method for single-trial recording of somatosensory evoked potentials," J. Biomed. Eng., vol. 15, pp. 257-262, 1993.

[18] J. P. C. De Weerd, "A posteriori time-varying filtering of averaged evoked potential I: Introduction and conceptual basis," Biol. Cybern., vol. 41, pp. 211-222, 1981

[19] A. Uncini, M. Marchesi, G. Orlandi, and F. Piazza, "Nonlinear adaptive filter using neural network," in Proc. Int. Conf. Neural Network, vol. 1, Paris, France, 1990, p. 158.

[20] M. V. Shirvaikar and M. M. Trivedi, "A neural network filter to detect small targets in high clutter backgrounds," IEEE Trans. Neural Network, vol. 6, pp. 252-257, Jan. 1995.

[21] E. J. Hartman, J. D. Keeler, and J. M. Kowalski, "Layered neural networks with Gaussian hidden units as universal approximation," Neural Computation, vol. 2, pp. 210-215, 1990.

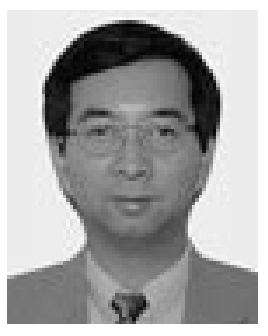

Wei Qiu (S'89-M'94) received the B.Eng. degree in biomedical engineering from Zhejiang University, China, in 1984, and M.Sc. and Ph.D. degrees in electronic engineering from South China University of Technology, China, in 1991 and 1994 respectively.

He is currently Senior Research Scientist of Auditory Research Laboratories, State University of New York, Plattsburgh, NY. From 1994 to 1996, he was on the Faculty of the Department of Electronic and Communication Engineering, South China University of Technology, China. He was a Postdoctoral Research Fellow at Department of Electrical and Electronic Engineering, Hong Kong University from 1996 to 1998 . His research interests are in the area of digital signal processing and applications.

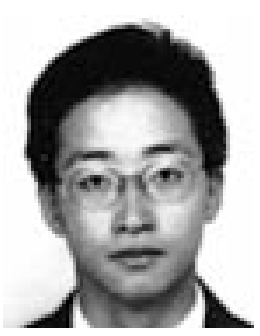

Kenneth S. M. Fung received the B.Eng. and Ph.D. degrees from The University of Hong Kong, Hong Kong, in 1994 and 1999, respectively.

Since 1999, he has been a Project Leader with the R \& D Department of ASM Assembly Automation Ltd., where he is in charge of the research team devoted to the development of computer vision algorithms. His research focuses on the application of artificial neural network filters. 


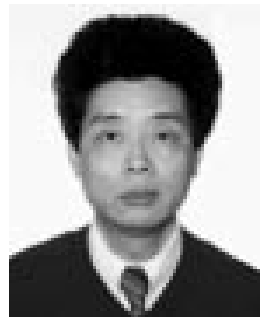

Francis H. Y. Chan (M'81) received the Ph.D. degree from Bristol University, Bristol, U.K.

$\mathrm{He}$ joined the Electrical and Electronic Engineering Department, The University of Hong Kong in 1976. Currently, he is Professor and Director of the University of Hong Kong Biomedical Engineering Research Centre, Hong Kong, coordinating biomedical engineering research. His research interest is in biomedical signal processing.

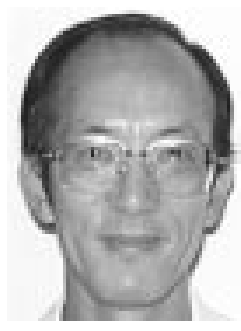

F. K. Lam obtained the B.Sc. degree in engineering from the University of Hong Kong, Hong Kong, and the M.Sc. and Ph.D. degrees from Loughborough University, Loughborough, U.K.

After working in Britain, he returned to Hong Kong University and now is Deputy Head of Department. His specialization is in signal processing and communication.

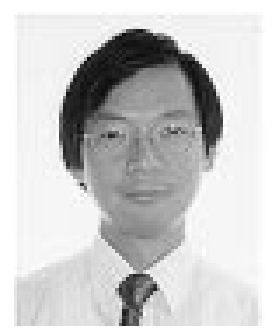

Paul W. F. Poon received the B.Sc. degree in biology from the Chinese University of Hong Kong, Hong Kong, in 1972, the M.Phil. degree in physiology from the University of Hong Kong, Hong Kong, in 1974, and the $\mathrm{Ph} . \mathrm{D}$. degree in neuroscience from Indiana University, Bloomington, in 1979.

After serving as a Lecturer at the Department of Physiology, University of Hong Kong, he moved to Taiwan in 1994, and is currently Professor and Chair in the Department of Electrical Engineering at the Imperial College, Cambridge University, and the University of Wisconsin. His interests in research include neural plasticity of the newborn, speech sound coding, evoked potential, neural imaging, and biomedical signal processing.

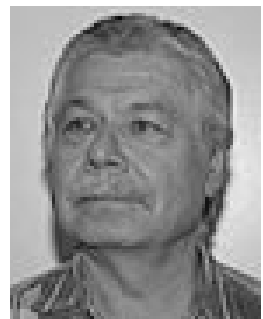

Roger P. Hamernik received the B.S., M.S., and Ph.D. degrees in mechanical engineering from Syracuse University, Syracuse, NY, in 1963, 1967, and 1970 respectively.

$\mathrm{He}$ is currently Professor of Physics, and Speech and Hearing and Director of the Auditory Research Laboratories, State University of New York, Plattsburgh. From 1980 to 1985, he was a Professor of Callier Certer for Communication Disorders, University of Texas at Dallas. His research interests are in the area of auditory sciences.

Dr. Hamernik is a Fellow of Acoustical Society of America. 